\title{
Do We Need Alternative Screening Approaches for Cervical Cancer During Covid-19 Pandemic?
}

\author{
Mertihan Kurdoğlu* ${ }^{*}$, Arash Khaki ${ }^{\circledR}$
}

$\longrightarrow$ ancer screening is very important for the women's health since it may allow the detection of not only precancerous lesions but also the cancers in their earliest stages, before the symptoms appear. However, the screening is not available for all types of cancer and has been highly recommended for cervical, breast, lung and colorectal cancers in certain ages and risk groups.

Coronavirus disease 2019 (COVID-19) pandemic had a negative impact on cancer screening by making the access to preventive care facilities difficult. It has been stated that during COVID-19 pandemic between March and July 2020, particularly the women and individuals aged 30 to 49 years have postponed cancer screenings in Germany (1).

Sharp decreases especially in the use of cervical cancer screening programs (2) may have led some cervical intraepithelial lesions to stay undetected in a potentially treatable stage of the cancerous progression. A two-month screening lock-down in Slovenia between March 12 and May 8, 2020 resulted in an 92\% epidemic deficit of screening, as well as $70 \%$ and $68 \%$ decrease in follow-up and human papilloma virus (HPV) triage tests, respectively (3). During the stay-at-home order in California, approximately $80 \%$ decrease in cervical cancer screening rates compared with baseline was observed in the Kaiser Permanente Southern California (KPSC) network including 1.5 million women (4). National data in Italy showed that beside the lock-down, the significant reduction in the number of screening tests was also related to the fear of COVID-19, which resulted in the suspension of booked screenings and the reduction in adherence to screenings (5).

The effects of disruption of cervical screening in COVID-19 pandemic can be mitigated by several approaches like HPV-based screening conducted from self-collected specimens. Such a reliable screening method may also allow to extend the screening intervals with longer reassurance against cervical cancer if it yields a negative result. The testing supported by telehealth

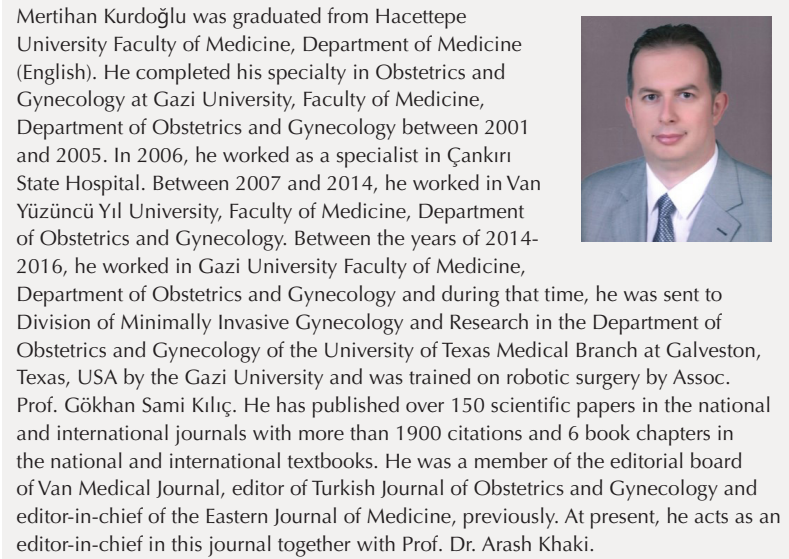

might further contribute to reduce the risk of COVID-19 exposure and patient-provider contact $(6,7)$.

As a result, to reduce human contact and curtail travel, HPV self-sampling may be a reasonable alternative screening approach for cervical cancer during COVID-19 pandemic.

Ethical Issues

Not applicable.

Conflict of Interests

None.

Authors' contributions

MK and AK: concept and design. MK: literature search and writing of the draft. AK: editing. Both authors read and approved the study.

\section{References}

1. Hajek A, De Bock F, Huebl L, Kretzler B, König H-H. Determinants of Postponed Cancer Screening During the COVID-19 Pandemic: Evidence from the Nationally Representative COVID-19 Snapshot Monitoring in Germany (COSMO). Risk Manag Healthc Policy. 2021;14:3003-3011. doi:10.2147/RMHP.S297326

2. Ahmadi M, Azizi Jalilian F, Dokhani N, Golparian M, Moradi Y. Evaluation of the prevalence of human papillomavirus in asymptomatic patients at the women's clinic in hamadan and comparing the 2 methods of Pap smear and PCR in detecting the virus. Int J Womens Health Reprod Sci. 2020;8:232-235. doi:10.15296/ijwhr.2020.37

Received 3 September 2021, Accepted 24 September 2021, Available online 2 October 2021

${ }^{1}$ Department of Obstetrics and Gynecology, Kırıkkale University Faculty of Medicine, Kırıkkale, Turkey. ${ }^{2}$ Clinical Research Development Unit of Tabriz Valiasr Hospital, Tabriz University of Medical Sciences, Tabriz, Iran.

*Corresponding Author: Mertihan Kurdoğlu, Tel: +90 3183335000 (Internal: 5215), Email: mkurdoglu@kku.edu.tr 
3. Ivanuš U, Jerman T, Gašper Oblak U, et al. The impact of the COVID-19 pandemic on organised cervical cancer screening: The first results of the Slovenian cervical screening programme and registry. Lancet Reg Health Eur. 2021;5:100101. doi:10.1016/j.lanepe.2021.100101

4. Miller MJ, Xu L, Qin J, et al. Impact of COVID-19 on Cervical Cancer Screening Rates Among Women Aged 21-65 Years in a Large Integrated Health Care System - Southern California, January 1-September 30, 2019, and January 1-September 30, 2020. MMWR Morbidity and Mortality Weekly Report. 2021;70:109-13. doi: 10.15585/mmwr. mm7004a1

5. Scioscia M, Noventa M, Palomba S, Laganà AS. Effect of the COVID-19 pandemic on oncology screenings: it is time to change course. BJOG.
2021. doi:10.1111/1471-0528.16857

6. Wentzensen N, Clarke MA, Perkins RB. Impact of COVID-19 on cervical cancer screening: Challenges and opportunities to improving resilience and reduce disparities. Prev Med. 2021;151:106596. doi:10.1016/j.ypmed.2021.106596

7. Ray C, Mandal R, Chatterjee P. Change in practice in cervical cancer screening during covid 19 pandemic. Abstracts of 17th World Congress of the International Federation of Colposcopy and Cervical PathologyIFCPC 2021. Indian Journal of Gynecologic Oncology 2021;19(4). doi: 10.1007/s40944-021-00548-2

(C) 2021 The Author(s); This is an open-access article distributed under the terms of the Creative Commons Attribution License (http:// creativecommons.org/licenses/by/4.0), which permits unrestricted use, distribution, and reproduction in any medium, provided the original work is properly cited. 